\title{
Exact treatment of time-dependent axisymmetric gravitation
}

\author{
by \\ P.C. Waylen \\ Department of Mathematics, University of Canterbury \\ Christchurch, New Zealand
}

No. 86

April, 1993

Published in Proc. R. Soc. Lond. A (March, 1993).

This Report contains also an appendix, not included in that paper. 


\title{
Exact treatment of time-dependent axisymmetric gravitation
}

\author{
by P.C. Waylen \\ Department of Mathematics, University of Canterbury, \\ Christchurch 1, New Zealand
}

\begin{abstract}
This paper extends an earlier treatment of time-dependent gravitational fields that are axially symmetric and nonrotating. From a consideration of the canonical solution of the Einstein vacuum field equations previously obtained as an axial expansion, a new method has been found that now provides the exact solution, whenever a certain generative key function $\chi(t, z)$ is known.
\end{abstract}




\section{Introduction}

Time-dependent, axisymmetric, nonrotating space-times that are solutions of Einstein's vacuum gravitation equations are currently undergoing careful scrutiny. Extensive numerical investigations of them are ongoing (see, for instance, Abrahams et al 1992), and the global asymptotic structures of examples like the one of Bonnor \& Swaminarayan (1964) have been analysed by Bič́k \& Schmidt (1989). Hence it now appears timely to produce new analytic examples of such space-times, both for direct use in the study of strong gravitational fields, and as test solutions for the numerical codes.

Attempts at finding exact solutions meet with the difficulty that Einstein's system of nonlinear partial differential equations is, for the time-dependent case, especially awkward. To help overcome this, the powerful method of reducing differential equations by similarity means (following Sophus Lie) has been extended and applied to gravitational theory, for example, by Harrison \& Estabrook (1971) and Stephani (1991). The latter paper uses the (complex) Ernst potential, and the paper's conclusion states, referring to the complicated nature of the systems of equations derived: "No general way of dealing with them is known; new techniques have to be developed."

The new feature of the present approach is that, rather than base the treatment on the Einstein field equations alone, we base it, instead, mainly on the canonical general solution of these equations, known already in the form of an axial series expansion (Waylen 1987). This approach effectively simplifies the problem to one of summing a series solution so as to get its closed form. A further bonus is that, owing to the prior existence of the axial series solution, the set of differential equations that will need solving in each case will automatically be integrable.

We shall soon see that those time-dependent solutions of Einstein's equations which may be obtained exactly, at least by the means of similarity reduction, are those solutions for which a certain function $\chi(t, z)$ can be determined from (7).

\section{Canonical solution using axial expansions}

Waylen (1987) has shown that, by using a sequence of coordinate transformations, it is possible to bring the general line element that satisfies Einstein's equations into a canonical form

$$
d s^{2}=A d t^{2}+2 B d t d z-C d z^{2}-\left(A C+B^{2}\right) d \rho^{2}-\rho^{2} d \phi^{2}
$$

where the functions $A, B, C$ depend on $t, z, \rho$. This general solution possesses, near the axis $\rho=0$, a convergent axial expansion

$$
\left.\begin{array}{l}
A=a\left\{1+\frac{1}{4}\left[\left(a^{-1}\right)_{, t t}-a, z z\right] \rho^{2}+O\left(\rho^{4}\right)\right\}, \\
C=a^{-1}\left\{1+\frac{1}{4}\left[\left(a^{-1}\right)_{, t t}-a, z z\right] \rho^{2}+O\left(\rho^{4}\right)\right\}, \\
B=0+0+O\left(\rho^{4}\right),
\end{array}\right\}
$$


with

$$
A C+B^{2}=1+\frac{1}{2}\left[\left(a^{-1}\right)_{, t t}-a_{, z z}\right] \rho^{2}+O\left(\rho^{4}\right),
$$

showing clearly that it does obey the necessary condition of regularity along the relevant segment of axis. This solution is determined completely by its arbitrary axial generating function $a(t, z)$.

Schwarzschild's static solution in these canonical coordinates is given by

$$
A=1-2 m / r, C=(1-2 m / r)^{-1}\left(1-2 m \rho^{2} / r^{3}\right)^{-1}, B=0,
$$

where $r$ may be obtained in terms of $z$ and $\rho$ from the equations of transformation

$$
z=(r-2 m) \cos \theta, \quad \rho=r \sin \theta \text {, }
$$

for which $\partial(z, \rho) / \partial(r, \theta)=r-2 m \sin ^{2} \theta$, being positive for $r>2 m$. We thus see that the axial generator of this solution is

$$
a=\left(1+\frac{2 m}{|z|}\right)^{-1}=1-\frac{2 m}{|z|}+O\left(\frac{1}{z^{2}}\right)
$$

displaying also its axial asymptotic behaviour for later reference.

\section{Form of the exact solutions}

To get exact solutions, it is more convenient first to express the generating function $a$ in terms of an alternative generative function $\chi(t, z)$, thus

$$
a=(-\chi, t t)^{1 / 2} /(\chi, z z)^{1 / 2}
$$

where, in particular, this function for flat space-time is

$$
\chi=k\left(z^{2}-t^{2}\right) \Rightarrow a=1
$$

with $k$ constant.

When subjected to a certain restriction (7), $\chi$ can be used to construct the exact forms of $A, B, C$, namely

$$
\left.\begin{array}{l}
A=a\left\{L+\left(1-f^{2}\right)^{-1}\left[2 f M+\left(1+f^{2}\right) N\right]\right\}, \\
C=a^{-1}\left\{L-\left(1-f^{2}\right)^{-1}\left[2 f M+\left(1+f^{2}\right) N\right]\right\}, \\
B=\left(1-f^{2}\right)^{-1}\left[\left(1+f^{2}\right) M+2 f N\right],
\end{array}\right\}
$$

with

$$
A C+B^{2}=L^{2}+M^{2}-N^{2} .
$$

The functions $L, M, N$ that appear here will at first be unknown functions of their two arguments,

$$
L\left(U, V \rho^{2}\right), \quad M\left(U, V \rho^{2}\right), \quad N\left(U, V \rho^{2}\right) .
$$


The generator $a$ is now regarded as being defined by (4), and the other functions appearing are defined by

$$
\begin{aligned}
f(t, z) & =a\left(\chi_{, z}+2 K t\right) /\left(\chi_{, t}-2 K z\right), \\
U(t, z) & =\left[a^{-1}\left(\chi_{, t}-2 K z\right)\right]_{, t}-\left[a\left(\chi_{, z}+2 K t\right)\right]_{, z}, \\
V(t, z) & =\left(a^{-1}\right)_{, t t}-a_{, z z},
\end{aligned}
$$

where $K$ is a dimensional constant. (Note that $V=0$ if the space-time is flat.)

These exact forms (6) of $A, B, C$ enable Einstein's field equations for the line element (1) to be satisfied for different functions $\chi$ by subsequently making a compatible choice of $L, M, N$. The condition that guarantees that this shall always be possible is that $\chi$ shall be some solution of the equation

$$
\begin{aligned}
4 \chi_{, t t} \chi_{, t z} \chi_{, z z} & +\left(\chi_{, t}-2 K z\right)\left[\chi_{, t t} \chi, z z z-\chi_{, z z} \chi_{, t t z}\right] \\
& +(\chi, z+2 K t)\left[\chi_{, z z} \chi_{, t t t}-\chi_{, t t} \chi_{, t z z}\right]=0 .
\end{aligned}
$$

We now stipulate that $\chi$ always will be such a solution and shall henceforth refer to it as the key function.

The general key function will involve, in some manner, three arbitrary elements, each one being a function with one argument. Thus if, for example, one formally expands this key function as a series in $t$,

$$
\chi=\chi_{0}(z)+\chi_{1}(z) t+\chi_{2}(z) t^{2}+O\left(t^{3}\right)
$$

one finds that the three leading coefficients are left arbitrary by (7), but that all the succeeding ones are uniquely determined.

\section{Determination of the unknown functions $L, M, N$}

From an exact key function, one can immediately construct the functions $a, f, U, V$, so that what remains to complete the construction of the exact solution (6) for $A, B, C$ is to solve for the functions $L, M, N$. In this section we shall briefly describe the solution procedure, and in the next section we shall work through an example.

After the substitution of (6) into the field equations, one puts

$$
U=u, \quad V \rho^{2}=v
$$

The latter abbreviation has the effect of removing $\rho$ from the field equations and inserting $v$. The former abbreviation equation, when solved algebraically for either $t$ or $z$, or, if more convenient, some particular combination of them, removes this coordinate or combination and inserts $u$. 
Thus one has the field equations now expressed in terms of the independent variables $u, v$ and some other one, which let us denote by $\zeta$.

In this new form, the field equations (actually their numerators) may always be satisfied by equating to zero the coefficients of all terms in $\zeta$ that are linearly independent. This will yield a set of partial differential equations in $u$ and $v$ alone, which must then be satisfied by the functions

$$
L(u, v), \quad M(u, v), \quad N(u, v)
$$

(Different key functions will of course give rise to different such sets of equations.)

Automatically, this set of equations will be integrable and, furthermore, it will always admit an axial series solution having precisely the form

$$
\left.\begin{array}{l}
L=1+\frac{1}{4} v+L_{2}(u) v^{2}+L_{3}(u) v^{3}+O\left(v^{4}\right), \\
M=0+0+M_{2}(u) v^{2}+M_{3}(u) v^{3}+O\left(v^{4}\right), \\
N=0+0+N_{2}(u) v^{2}+N_{3}(u) v^{3}+O\left(v^{4}\right) .
\end{array}\right\}
$$

The coefficients will be determinable by simple algebra.

This series solution for $L, M, N$ should always be obtained first. It will suggest the form of the exact solution, and thus shorten the process of elimination leading to the exact solution. It can also be employed during this process to resolve any ambiguities of sign arising from the taking of roots, and to assign the necessary values to the constants of integration.

\section{Example of an exact solution}

To serve as our illustration, we shall select the relevant key function that is the next simplest one to (5). This is

$$
\chi=\frac{1}{3} K\left(z^{2}-t^{2}\right)+\frac{4}{3} K m\left\{\zeta \ln \left[K \zeta+K\left(\zeta^{2}+4 m^{2}\right)^{1 / 2}\right]-\left(\zeta^{2}+4 m^{2}\right)^{1 / 2}\right\}
$$

where we have written $z-t=\zeta$.

From this, we obtain, on also writing $\left(\zeta^{2}+4 m^{2}\right)^{1 / 2}=s$,

$$
a=\left(\frac{s-2 m}{s+2 m}\right)^{1 / 2}=1-\frac{2 m}{|z|}+O\left(\frac{1}{z^{2}}\right),
$$

and

$$
\begin{aligned}
f & =-\left(\frac{s-2 m}{s+2 m}\right)^{1 / 2} \cdot \frac{4 t+\zeta+2 m \ln [K(\zeta+s)]}{4 t+3 \zeta+2 m \ln [K(\zeta+s)]} \\
U & =-\frac{4}{3} K\left\{\zeta^{3}+4 m(2 t+\zeta) s+4 m^{2}[\zeta+s \ln [K(\zeta+s)]]\right\} /\left(\zeta^{2} s\right), \\
V & =8 m / \zeta^{3} .
\end{aligned}
$$


Next, in order to secure the functions $L, M, N$, we substitute the resulting forms (6) into the field equations. Using $V \rho^{2}=v$, we see that $\rho^{2}$ should be replaced by $\left(\zeta^{3} / 8 m\right) v$, and by solving $U=u$, we get a value with which to replace $t$.

The field equations are thus now expressed in terms of $u, v, \zeta$. Hence we readily obtain the reduced set of equations that must be satisfied by $L, M, N$.

Upon substituting the form (9) of the axial series into this set of equations, we get for this case the unique series solution

$$
\left.\begin{array}{l}
L=1+\frac{1}{4} v+\frac{9}{32} v^{2}+\frac{47}{128} v^{3}+O\left(v^{4}\right), \\
M=\frac{24 K u}{9 u^{2}-16 K^{2}}\left[\frac{3}{32} v^{2}+\frac{23}{128} v^{3}+O\left(v^{4}\right)\right], \\
N=\frac{9 u^{2}+16 K^{2}}{9 u^{2}-16 K^{2}}\left[\frac{3}{32} v^{2}+\frac{28}{128} v^{3}+O\left(v^{4}\right)\right],
\end{array}\right\}
$$

which suggests that the exact solution will possess the form

$$
L=X(v), \quad M=\frac{24 K u}{9 u^{2}-16 K^{2}} Y(v), \quad N=\frac{9 u^{2}+16 K^{2}}{9 u^{2}-16 K^{2}} Y(v) .
$$

As a result of a straightforward process of elimination between the resulting ordinary differential equations for $X(v)$ and $Y(v)$, we find that by putting

$$
X=\frac{1}{2} \frac{\left(1+3 Z^{2}-2 Z^{3}\right)}{Z(3-2 Z)}, \quad Y=\frac{1}{2} \frac{(1+2 Z)(1-Z)^{2}}{Z(3-2 Z)},
$$

the original set of second-order partial differential equations for $L, M, N$ is reduced finally to a single first-order ordinary differential equation for $Z(v)$,

$$
v(9 v Z-8) Z^{\prime 2}+2(3 v Z-2) Z Z^{\prime}+Z^{3}=0 .
$$

The solution of this that coincides with (12) is

$$
(Z-1) / Z^{3}=\frac{1}{4} v \text {. }
$$

We have therefore now arrived at the exact solution of Einstein's field equations that is produced by the simple key function (10). This exact solution is

$$
\begin{aligned}
& A=\frac{a}{1-a^{2}}\left[1-a^{2} Z^{2}(3-2 Z)\right] /[Z(3-2 Z)], \\
& C=\frac{a^{-1}}{1-a^{2}}\left[Z^{2}(3-2 Z)-a^{2}\right] /[Z(3-2 Z)], \\
& B=\frac{-a}{1-a^{2}}(1+2 Z)(1-Z)^{2} /[Z(3-2 Z)],
\end{aligned}
$$

where $a$ is given by (11), $Z$ by (13), and $v=8 m \rho^{2} /(z-t)^{3}$.

Along the axis $\rho=0$, we have

$$
v=0 \Rightarrow Z=1 \Rightarrow\left\{A=a, C=a^{-1}, B=0\right\},
$$

which is in accordance with (2). 


\section{Conclusion}

A method has been found for transforming key functions $\chi(t, z)$ that satisfy (7) into exact solutions of Einstein's vacuum field equations.

Exact solutions of physical interest should probably possess a generating function $a(t, z)$, following from (4), that emulates the leading axial asymptotic behaviour (3) of Schwarzschild's solution, and which in addition, again like Schwarzschild's solution, is such that $a=0$ at those points on the axis where masses are located. The problem of obtaining such exact solutions has been reduced in this paper to one of obtaining the exact forms of the appropriate key functions.

Of particular interest would be the key function whose expansion (8) is

$$
\chi=\frac{1}{4} K\left(z^{2}-t^{2}\right)+2 K(m / z)(z-m)(z-t) \ln \left|z / z_{0}\right|+O\left(t^{3}\right)
$$

because its consequential solution of the field equations may be expected to resemble a timedependent, axisymmetric version of Schwarzschild's solution.

\section{References}

Abrahams, A., Bernstein, D., Hobill, D., Seidel, E. \& Smarr, L. 1992 Phys. Rev. D 45, $3544-3558$.

Bičák, J. \& Schmidt, B. 1989 Phys. Rev. D 40, 1827-1853.

Bonnor, W.B. \& Swaminarayan, N.S. 1964 Z. Phys. 177, 240-256.

Harrison, B.K. \& Estabrook, F.B. 1971 J. Math. Phys. 20, 1486-1489.

Stephani, H. 1991 J. Math. Phys. 32, 1552-1555.

Waylen, P.C. 1987 Proc. R. Soc. Lond. A 411, 49-57. 


\section{Appendix}

In this appendix we give another example of an exact solution of the field equations $R_{\mu \nu}=0$. It has been obtained by putting $K=0$ and taking as the key function,

$$
\chi=z^{2 / 3}\left(t^{2}+T^{2}\right)
$$

where $T$ is a constant. (This easy choice does not lead to a physical solution.)

We find for this case that

$$
L=\frac{1}{2}(P+Q), \quad M=0, \quad N=\frac{1}{2}(P-Q),
$$

where

$$
P=2\left(3 Q^{2}-2\right)^{3 / 4} /\left[Q^{1 / 2}\left(3 Q^{2}-1\right)\right] .
$$

The function $Q(v)$ is to be determined from

$$
Q\left(Q^{2}-1\right)=\frac{1}{2} v,
$$

so that we have $Q=1$ along the axis $\rho=0$.

In this case $\quad v=\frac{1}{3} T^{2} \rho^{2} /\left[z\left(t^{2}+T^{2}\right)^{3 / 2}\right]$,

and the solution that follows is

$$
\begin{aligned}
& A=3 z\left[Q\left(t^{2}+T^{2}\right)-P t^{2}\right] /\left[T^{2}\left(t^{2}+T^{2}\right)^{1 / 2}\right], \\
& C=\frac{1}{3}\left(t^{2}+T^{2}\right)^{1 / 2}\left[P\left(t^{2}+T^{2}\right)-Q t^{2}\right] /\left(z T^{2}\right), \\
& B=t\left(t^{2}+T^{2}\right)^{1 / 2}(Q-P) / T^{2} .
\end{aligned}
$$

It is not flat, because $V=v / \rho^{2} \not \equiv 0$. 